\title{
Curiosity vs. Control: Impacts of Training on Performance of Teams Working with Robots
}

\author{
Sangseok You \\ School of Information \\ University of Michigan \\ Ann Arbor, MI 48109, USA \\ sangyou@umich.edu \\ Lionel P. Robert Jr. \\ School of Information \\ University of Michigan \\ Ann Arbor, MI 48109, USA \\ Iprobert@umich.edu
}

Abstract

Training robot operators is one approach to promoting better performance in teams working with robots. Yet, training does not always result in better performance. This study conducts a preliminary analysis of why by examining two psychological states of use: control and curiosity. An experimental study involving 30 teams two humans and two robots employing robots was conducted. Results showed that training minimized the negative impacts of curiosity and heightened the positive impacts of control on task involving the use of a robot.

Permission to make digital or hard copies of part or all of this work for personal or classroom use is granted without fee provided that copies are not made or distributed for profit or commercial advantage and that copies bear this notice and the full citation on the first page. Copyrights for third-party components of this work must be honored. For all other for third-party components of this

Copyright is held by the owner/author(s).

CSCW '16 Companion, February 27 - March 02, 2016, San Francisco, CA USA

ACM 978-1-4503-3950-6/16/02

http://dx.doi.org/10.1145/2818052.2869121

\author{
Author Keywords \\ teams; curiosity; control; exploration; exploitation; \\ performance; human-robot collaboration
}

\section{ACM Classification Keywords}

H.5.2 [User Interfaces and Presentation (e.g., HCI)]: Theory and Methods; H.1.2 [Models and Principles]:

User/Machine Systems - human factors.

\section{Training in Teams Working with Robots}

Robots are increasingly adopted in teams in many areas [6]. For instance, search and rescue teams deploy remote-controlled robots to save human lives [1]. In such teams, the ability to use robots is central to their success $[1,2]$. Despite the importance of this topic, there is much we do not know about facilitating better performance in teams working with robots $[2,7]$.

Training robot operators has been one approach to promoting better performance in such teams [4]. For example, training in teams working with robots has led to better coordination and higher level of trust [4]. Unfortunately, training does not always lead to increases in performance $[4,8]$. We believe that understanding when training is likely to be effective or not is vital to promoting better performance in teams working with robots. 
There are two psychological states associated with both training and performance: exploration and exploitation $[3,5]$. Exploration as it pertains to technology use is often associated with curiosity. Curiosity as a psychological state can lead individuals to play with the technology and discover new features [5]. Exploitation is often associated with the psychological state of control as it relates to technology [5]. In the short term, curiosity and control has been found to have opposite relationship with performance. Curiosity decreases short-term performance with technology, whereas control increases short-term performance [3].

We propose that the effectiveness of training is likely to depend on the user's psychological state while employing the system. Both curiosity and control have been associated with training and performance $[3,5]$. Therefore, we propose that these two psychological states are essential to understanding when training is more or less effective in increasing performance in teams working with robots.

The goal of this preliminary study is to investigate conditions which help determine when training becomes more or less effective in increasing performance. To do this, we conducted a lab-based experiment, in which we examined the impacts of training on performance in 30 teams of two humans working with two robots depending on their state of curiosity and control. Results of this study generally shows evidence of influence of curiosity and control on the impacts of training. This study will open a new opportunity of studying teamwork for scholars in CSCW as well as teams working with robots.

\section{Moderation Effects of Curiosity and Control}

\section{in the Impacts of Training}

We propose that the impacts of training on

performance will be moderated by the level of curiosity and control in teams working with robots. That is, although training is known, in general, to increase performance, the impacts can appear differently based on the level of curiosity and control during the training.

When teams perceive higher level of curiosity of robots, they will likely explore features of robots through risk taking, innovation, and flexibility [3]. These behaviors will foster team's engagement in the training and in turn increase overall task performance. Thus, training will be more effective in increasing performance when the level of curiosity is high.

H1(a \& b): Training will increase (a) individual and (b) team performance of teams working with robots, when teams perceive higher level of curiosity than lower.

When feeling in control, teams will likely perform the task with behaviors including refinement, production, and efficiency in mind [3]. Such behaviors are focused on achieving their goal, which will lead to better performance. However, for those who do feel lower level of control, training will lead them to engage with effective ways of using the robots to fulfill the task.

H2(a \& b): Training will increase (a) individual and (b) team performance of teams working with robots, 
when teams perceive lower level of control than higher.

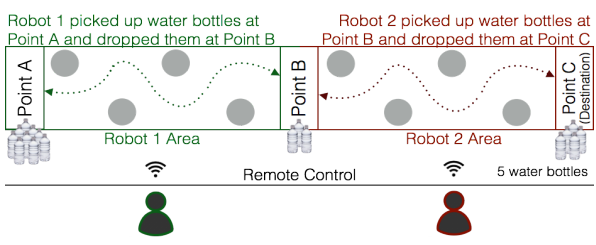

Figure 1. Experimental Task Setting

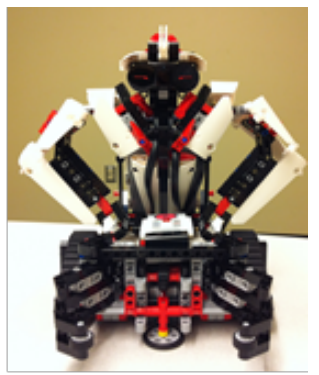

Figure 2. A remote-controlled robot used for the experiment

\section{Method}

Sixty individuals were recruited for an experiment at a large university in US (39 females, $M_{\text {age }}=22.88$, and $\left.\mathrm{SD}_{\text {age }}=4.51\right)$. Individual participants were randomly assigned to a team of two humans and two robots ( $\mathrm{N}=$ 30). Each team was also randomly assigned to either training or no-training condition.

\section{Experimental Task}

The experimental task was to move five plastic water bottles from one point to another point by controlling their robots. Teams were told to finish the delivery of water bottles from point $A$ to $C$ as quickly as possible. The task was designed to be team-based collaboration, but consisted of two individual subparts. In each team, one person (Person 1) delivered water bottles from point $A$ to $B$, while the other person (Person 2) delivered from point $B$ to $C$. The task was performed in a large room, in which teams were allowed to communicate and strategize to complete the task.

\section{Robots}

Two identical robots were used for each team in the experiment (Figure 2). The robots were built with Lego Mindstorms EV3. The robots were controlled by infrared remote controllers and capable of gripping the plastic water bottles, holding them while moving, and releasing them.

\section{Manipulation of Training}

Manipulation of training had two levels: no-training and training. In no-training condition, participants did not have an opportunity to play with their robots before the team task. In training condition, two trial runs of the team task without timing were given to individuals in teams before the main team task.

\section{Measures}

Curiosity and control were measured using a 5-point Likert scale ranging from 1 for "strongly disagree" to 5 for "strongly agree" and aggregated to the team-level after the interaction. Both of the constructs indicated Cronbach's $\alpha$ higher than .8 and ICC(1) above .1 and were standardized.

- Curiosity was measured using an index of three items adopted from [5]. An example was "Using the robot excited my curiosity."

- Control was measured using an index of two items from [5]. One item was "When using the robot, I felt in control."

Individual performance was obtained by averaging the time it took for individuals to deliver five water bottles from one point to another point. Team performance was measured by total time to finish deliver five water bottles from point $\mathrm{A}$ to $\mathrm{C}$.

\section{Results}

Results of multilevel analyses showed that there were interaction effects between presence of training, curiosity and control, respectively. As hypothesized in $\mathrm{H} 1$, training increased individual performance when the level of curiosity was high, whereas it had no impact when it was low $(\beta=-13.44, p<.001)$ (Figure 3 ). However, the interaction effect was not found with team performance $(\beta=-15.38, p=.35)$. For hypothesis 2 , training was found to increase team performance to a greater degree when the level of control was low than when control was high $(\beta=35.48$, 


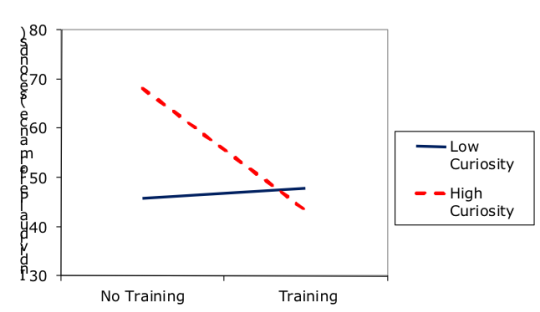

Figure 3. Two-way interaction between curiosity and training on individual performance

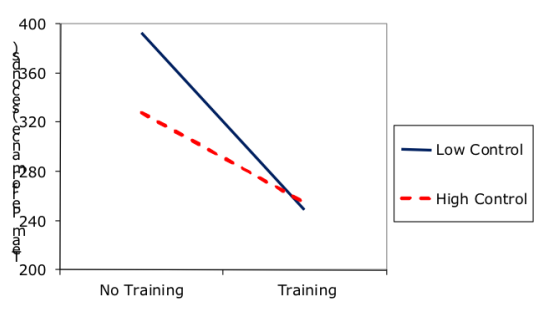

Figure 4. Two-way interaction between control and training on team performance $p<.05)$ (Figure 4$)$. No interaction effect was found with individual performance $(\beta=4.78, p=.22)$

\section{Implications for Theory and Practice}

The preliminary results have potential implications for theory and practice of teams working with robots. First, research should examine impacts of training in teams working with robots. We found that training caused different consequences in individual and team performance depending on the level of curiosity and control. This suggests that the impact of training can be altered by psychological states of individuals in the training. However, much more research is needed to find more circumstances that can determine impacts of training in teams working with robots.

Second, our preliminary results may signal a need to revisit some of our prior literature on exploration and exploitation of technology. Currently, it is assumed that curiosity is bad for short term performance and good for long term performance [3]. This leads to the discussions around the trade-off between short vs. long term performance. Our results suggest that this may not be the case. When individuals are trained, curiosity does not have to lead to lower performance. Therefore, it is possible to encourage curiosity in short term without suffering performance losses while stil incurring the potential long term performance gains.

\section{Conclusion}

Training is important to understanding performance in teams working with robots. However, little is known on when training is more or less effective in teams working with robots. Our preliminary results revealed different impacts of training on using robots based on curiosity and control. This study has potential to motivate more research of teams working with robots on this topic.

\section{References}

[1] Burke, J.L., Murphy, R.R., Coovert, M.D., and Riddle, D.L. Moonlight in miami: Field study of humanrobot interaction in the context of an urban search and rescue disaster response training exercise. HumanComputer Interaction 19, 1-2 (2004), 85-116.

[2] Duysburgh, P., Elprama, S. A., \& Jacobs, A. Exploring the social-technological gap in telesurgery: collaboration within distributed or teams. Proc. of CSCW2014. ACM (2014). 1537-1548

[3] Kane, G.C. and Alavi, M. Information technology and organizational learning: An investigation of exploration and exploitation processes. Organization Science 18, 5 (2007), 796-812.

[4] Nikolaidis, S. and Shah, J. Human-robot Crosstraining: Computational Formulation, Modeling and Evaluation of a Human Team Training Strategy. Proc. of HRI2013, IEEE Press (2013), 33-40.

[5] Reychav, I. and Wu, D. Are your users actively involved? A cognitive absorption perspective in mobile training. Computers in Human Behavior 44, (2015), 335-346.

[6] Robert, L.P. and You, S. Human-Robot Interaction in Groups: Theory, Method, and Design for Robots in Groups. Proc. of GROUP2014, ACM (2014), 310-312.

[7] Robert, L.P. and You, S. Subgroup Formation in Teams Working with Robots. Proc. CHIEA2015, ACM (2015), 2097-2102.

[8] Salas, E., Tannenbaum, S.I., Kraiger, K., and Smith-Jentsch, K.A. The science of training and development in organizations: What matters in practice. Psychological science in the public interest 13 2 (2012), 74-101. 